\title{
Fatty amides synthesized from vegetable oil as extractant of molybdenum(VI)
}

\begin{abstract}
In this study, fatty amides (FAs) synthesized from palm olein were used to extract and separate Mo(VI) from acidic media. Effects of various parameters upon the separation of $\mathrm{Mo}(\mathrm{VI})$ from $\mathrm{Co}(\mathrm{II}), \mathrm{Ni}(\mathrm{II}), \mathrm{Al}(\mathrm{III})$ and $\mathrm{Mn}$ (II), including extractant concentration, metal ion concentration, contact time, diluent, and acidity, were investigated. It was found that Mo(VI) was successfully separated from the above commonly associated metal ions by stripping from the loaded organic phase. Different acidic and alkaline solutions were used. Ammonium hydroxide solution was an optimal. Extraction of Mo(VI) into the organic phase involved the formation of 1:3 complexes. This work presents the development of a low-cost and environmentally friendly extractant to recycle and recover molybdenum.
\end{abstract}

Keyword: Acidic media; Fatty amides; Molybdenum(VI). 